$\left(\begin{array}{llr}\text { Jpn. J. Hosp. Pharm } & \text { P } \\ \hline \text { 般 } & \text { 妾 } \\ 24(2) 130-135 & (1998)\end{array}\right)$

\title{
Evaluation of Clonazepam Pharmacokinetics in Epileptic Children Receiving Monotherapy or Polytherapy, and the Influences of Concomitant Antiepileptic Drugs on the Serum Concentration of Clonazepam
}

\author{
KAZURO IKAWA $\dagger^{1}$, NORIFUMI MORIKAWA $\uparrow^{1}$, NOBUOKI ESHIMA $\dagger^{2}$, \\ TATSURO IZUMI $\dagger^{3}$ and MASAHARU TAKEYAMA $\dagger^{1}$ \\ Department of Clinical Pharmacy $\dagger^{1}$, Oita Medical University \\ Department of Medical Information Analysis $\dagger^{2}$, Oita Medical University \\ Department of Pediatrics $\dagger^{3}$, Oita Medical University \\ $\left(\begin{array}{l}\text { Received April 21, } 1997 \\ \text { Accepted November 14, } 1997\end{array}\right)$
}

\begin{abstract}
We investigated the relationship between the steady-state serum concentration of clonazepam and the daily dose per weight in epileptic children under 12 years of age who were receiving chronic monotherapy or polytherapy. We also examined the effects of the concurrent administration of other antiepileptic drugs, including phenobarbital (PB), phenytoin (PHT), carbamazepine (CBZ), zonisamide (ZNS), and valproic acid (VPA), on the serum concentration of clonazepam by multiple linear regression analysis. The clonazepam concentration in the same daily dose and the concentration/dose ratio were lower in the polytherapy group than in the monotherapy group. The clonazepam concentration was noted to be positively proportional to the dose of clonazepam and was negatively affected by the addition of PB, PHT, and CBZ, but not of ZNS or VPA. The present results suggest that the differences of clonazepam pharmacokinetics in the monotherapy and polytherapy are associated with the effects of $\mathrm{PB}, \mathrm{PHT}$, and $\mathrm{CBZ}$ on the serum concentration of clonazepam.
\end{abstract}

Key words - clonazepam, drug interactions, serum concentrations, epileptic children, therapeutic drug monitoring

\section{Introduction}

Clonazepam effectively controls the various forms of epileptic seizures ${ }^{1)}$ and has been used as a wide-spectrum antiepileptic drug in Japan since 1980. Although data on pharmacokinetics are available after one-time or repeated administration ${ }^{2)}$, the pharmacokinetics of chronic therapy with clonazepam, especially in a large epileptic population, have not been extensively investigated. Some studies have reported a significant correlation between the concentration of clonazepam and the daily dose ${ }^{3,4}$, while others have shown a poor correlation ${ }^{5,6)}$. The discrepancy may be related to differences in the age of the patients studied, the concomitant use of other antiepileptic drugs, and differences in the interval between dosing and blood sampling ${ }^{7}$. We recently observed a significant 
correlation between the serum concentration of clonazepam and the daily dose per weight, and found that the patient age at which the concentration/daily dose of clonazepam (C/D) ratio showed significant changes was about 11 years ${ }^{8)}$.

Clonazepam is often used as adjunctive therapy, but rarely as a primary therapy, in patients with epilepsy. In spite of the polypharmaceutical nature of clonazepam therapy, the difference in clonazepam pharmacokinetics between monotherapy and polytherapy and the interaction between clonazepam and other antiepileptic drugs are still unclear ${ }^{1)}$. Most studies examining drug interactions investigated the interactions between clonazepam and only one additional antiepileptic drug.

In this study, we examined the relationship between the steady-state serum concentration of clonazepam and the daily dose per weight in epileptic children under 12 years of age who were receiving chronic monotherapy or polytherapy. We also studied the effects of concomitantly administered antiepileptic drugs on the serum concentration of clonazepam by multiple linear regression analysis.

\section{Materials and Methods}

\section{Subjects}

The study involved 147 pediatric inpatients or outpatients aged less than 12 years (75 males and 72 females)who had been treated at Oita Medical University Hospital during the past seven years. All subjects received clonazepam (Landsen powder or tablets, Sumitomo Pharmaceutical Co. Ltd., Osaka, Japan) as montherapy or polytherapy administered in two or three divided doses to control epileptic seizures. The blood samples were drawn from 7 to 14 hours after the preceding dose (not in the absorption phase of clonazepam). We collected one steady-state clonazepam concentration for each patient as long as the duration in the same combination of anticonvulsants exceeded two weeks. These data provided a total 164 concentrations. Patients in the polytherapy group were also receiving phenobarbital $(\mathrm{PB}, \mathrm{n}=9)$, phenytoin ( $\mathrm{PHT}, \mathrm{n}=36)$, carbamazepine ( $\mathrm{CBZ}, \mathrm{n}=43$ ), zonisamide (ZNS, $n=31$ ), and valproic acid (VPA, $n=112$ ). The number of patients receiving ethosuximide, primidone, benzodiazepines, and bromides was too small to permit analysis. The principal characteristics of the patient population studied are presented in Table 1.

Table 1. Subject Characteristics in the Clonazepam Monotherapy and Polytherapy Groups

Each value represents the mean \pm standard deviation.C/D, the serum concentration of clonazepam/ the clonazepam daily dose per weight.

\begin{tabular}{llc}
\hline & Monotherapy & Polytherapy \\
\cline { 2 - 3 } & & \\
\cline { 2 - 3 } Number of patients (male/female) & $17(7 / 10)$ & $130(68 / 62)$ \\
Number of observations & 17 & 147 \\
Age (years) & $5.18 \pm 5.16$ & $5.91 \pm 3.84$ \\
Weight $(\mathrm{kg})$ & $20.2 \pm 16.4$ & $20.6 \pm 13.3$ \\
Concentration in serum $(\mathrm{ng} / \mathrm{ml})$ & $25.6 \pm 11.2$ & $24.2 \pm 14.4$ \\
Daily dose $(\mu \mathrm{g} / \mathrm{kg})$ & $56.7 \pm 23.1$ & $88.0 \pm 53.3$ \\
C/D ratio $(\mathrm{ng} / \mathrm{ml} / \mu \mathrm{g} / \mathrm{kg})$ & $0.456 \pm 0.115$ & $0.318 \pm 0.150$ \\
Number of concomitant & - & $1.57 \pm 0.662$ \\
anticonvulsant (/observation) & & \\
\hline
\end{tabular}




\section{Measurement}

Serum samples were centrifuged and stored at $4^{\circ} \mathrm{C}$ until assayed. To determine the clonazepam concentration, serum samples $(1 \mathrm{ml})$ were extracted and subjected to reverse HPLC, according to a previously described method ${ }^{8)}$.

\section{Data analysis}

Data were analyzed by the following model for expressing the effect of addition of each concomitant anticonvulsant :

$$
C=\alpha+\beta_{1} D+\beta_{2} \mathrm{~PB}(01)+\beta_{3} \mathrm{PHT}(01)+\beta_{4} \mathrm{CBZ}(01)+\beta_{5} \mathrm{ZNS}(01)+\beta_{6} \mathrm{VPA}(01)+\varepsilon
$$

where $\mathrm{C}$ is the serum concentration of clonazepam $(\mathrm{ng} / \mathrm{ml}), \mathrm{D}$ is the clonazepam daily dose per weight $(\mu \mathrm{g} / \mathrm{kg})$, PB (01), PHT (01), CBZ (01), ZNS (01), and VPA (01) are the alternative dummy variables that represent the addition $(1)$ or the absence $(0)$ of the corresponding drug, e.g. $\mathrm{PB}(01)=\mathrm{PHT}(01)=\mathrm{CBZ}(01)=\mathrm{ZNS}(01)=\mathrm{VPA}(01)=0$ in the case of the monotherapy. $\alpha$ is a regression constant, $\beta$ is a regression coefficient of the corresponding drug, and $\varepsilon$ is an error term that is normally distributed with mean 0 and variance $\sigma^{2}$. The multiple linear regression analysis was performed by both the forward selection procedure and the backward elimination procedure with a packaged software program (SPSS ver. 6.1, SPSS Inc., Tokyo, Japan) on a NEC microcomputer (Tokyo, Japan). The significance of coefficients and constants was assessed by t-test, and the level of significance was 0.05 .

\section{Results and Discussion}

The steady-state serum concentration of clonazepam showed a correlation with the daily dose, suggesting the concentration in the same daily dose was lower in the polytherapy group than in the monotherapy group (Fig. 1). As summarized in Table 2, the linear regression analysis gave the

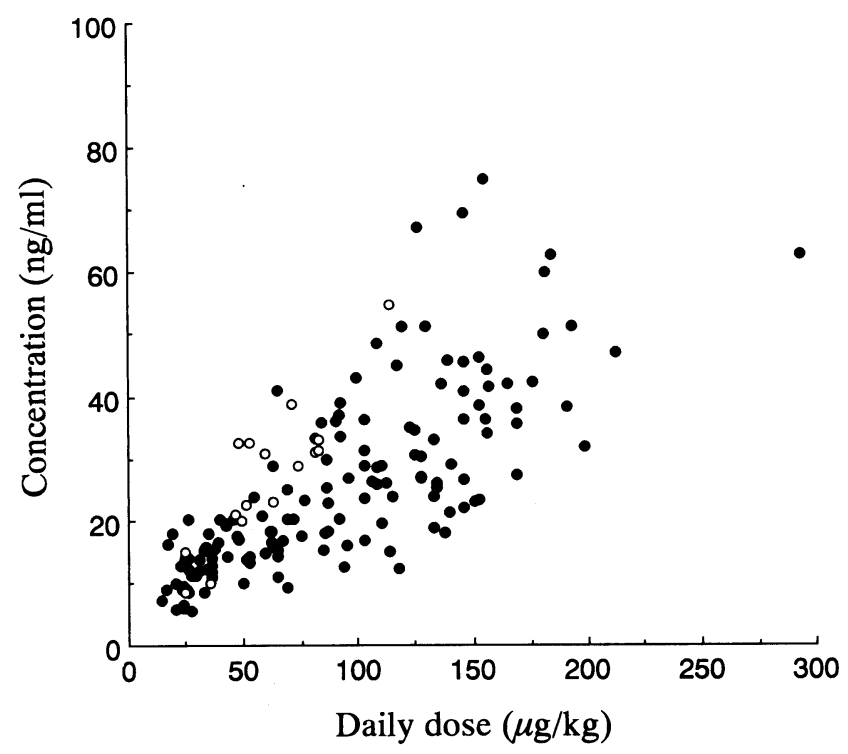

Fig. 1. Serum Concentrations of Clonazepam in Relation to the Daily Dose per Weight in the Monotherapy $(\bigcirc)$ and Polytherapy

(O) Groups 
Table 2. The Estomated Parameters of Final Linear Regression Model (1) for the Serum Concentration of Clonazepam

D, the clonazepam daily dose per weight; PB(01), PHT(01), and CBZ $(01)$, the alternative dummy variables that represent the addition (1) or the absence $(0)$ of the corresponding drug.

\begin{tabular}{lclllll}
\hline Variable & Estimated coefficient & Standard error & 95\% confidence interval & t statistic & $p$-value \\
\hline D & 0.217 & 0.0127 & $(0.192,0.242)$ & 17.0 & 0.0000 \\
PB(01) & -11.9 & 2.88 & $(-17.6,-6.23)$ & -4.14 & 0.0001 \\
PHT(01) & -7.72 & 1.57 & $(-10.8,-4.63)$ & -4.92 & 0.0000 \\
CBZ(01) & -5.58 & 1.50 & $(-8.54,-2.63)$ & -3.73 & 0.0003 \\
(Constant) & 9.81 & 1.32 & $(7.21,-12.4)$ & 7.43 & 0.0000 \\
\hline
\end{tabular}

following result :

$$
\begin{aligned}
& \mathrm{C}=0.217 \mathrm{D}-11.9 \mathrm{~PB}(01)-7.72 \operatorname{PHT}(01)-5.58 \mathrm{CBZ}(01)+9.81 \\
& \quad(\mathrm{R}=0.814, \mathrm{p}<0.01, \mathrm{n}=164)
\end{aligned}
$$

The serum concentration of clonazepam was positively proportional to the dose of clonazepam and was negatively affected by the addition of PB, PHT, and CBZ, but not of ZNS or VPA. The coefficient of determination $R^{2}$ was 0.663 because the multiple correlation coefficient $R$ was 0.814 , i. e. $66.3 \%$ of variance of data was explained by the final linear regression model (1). The relationship between the observed and final model-calculated concentrations of clonazepam is

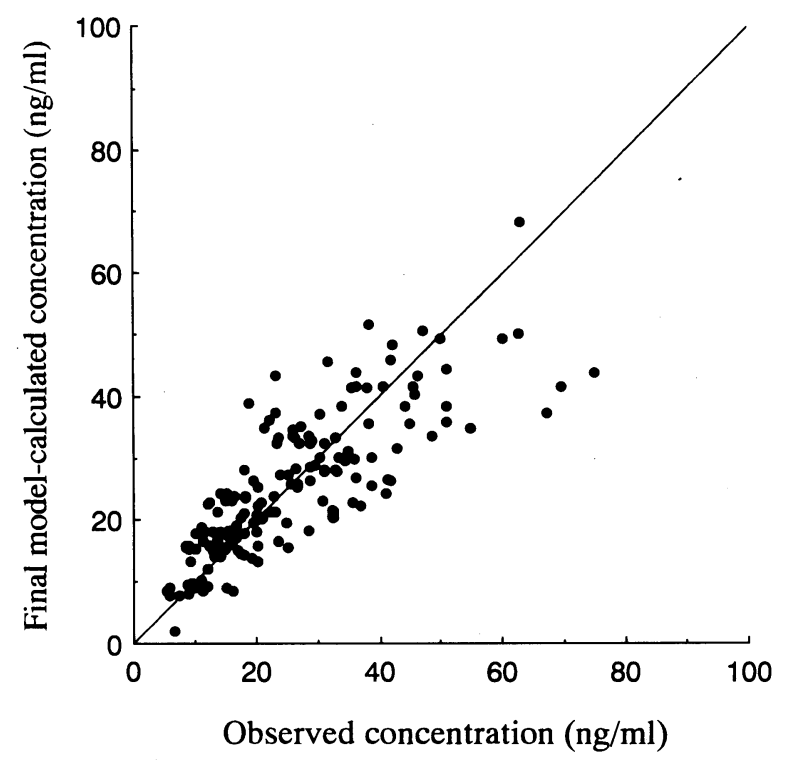

Fig. 2. Plot of Obsreved Serum Clonazepam Concentration vérsus Final Model-Calculated Concentration

The reference line represents that calculated concentration equals the observed one. 


\section{shown in Fig 2.}

Baruzzi et al. ${ }^{7)}$ have suggested that the correlation between the clonazepam level and the dose is affected by factors such as patient age, concomitantly administered antiepileptic drugs, and the interval between the preceding dose and blood sampling. In the present study, the clonazepam concentration, not in the absorption phase of clonazepam, was correlated with the daily dose in patients younger than 12 years of age. We previously found an age-related difference in the correlation between the clonazepam level and the daily dose in patients younger and older than 11 years of age ${ }^{8)}$. The present results showed that PB, PHT, and CBZ caused decreases in the clonazepam level in linear-combination mode, which may explain why the clonazepam concentration in the same daily dose and the $\mathrm{C} / \mathrm{D}$ ratio were lower in the polytherapy group than in the monotherapy group (Fig.1, Table 1).

The present results are consistent with the results of previous studies showing that PB, PHT, and CBZ alter the pharmacokinetics of a variety of drugs including anticonvulsants. $\mathrm{PHT}^{\left({ }^{10}\right.}$ and $\mathrm{PB}^{11)}$ have been found to reduce the concentration of clonazepam. Lai et al. ${ }^{12}$ reported that concurrent administration of $\mathrm{CBZ}$ reduces the plasma concentration of clonazepam in healthy volunteers, and Sunaoshi et al. ${ }^{13)}$ noted the clonazepam level increases when patients receiving combination therapy with clonazepam and CBZ are switched to clonazepam monotherapy. ZNS and VPA had no significant effect on the clonazepam concentration in the present study. Previous studies have also shown that VPA has no effect on the clonazepam concentration ${ }^{14,15)}$, but no direct evidence of the effect of ZNS on the clonazepam concentration is available.

The present results do not clarify the mechanisms of the interactions between clonazepam and other anticonvulsants. However, we speculate that the concomitant antiepileptic drugs may induce an enzyme that accelerates the metabolism of clonazepam. Animal studies have shown that $\mathrm{PB}^{16)}$ can induce the enzyme that mediates nitro reduction, a primary route of clonazepam metabolism. Previous studies have shown that the protein binding of clonazepam is not affected by concomitant use of $\mathrm{PB}^{10)}, \mathrm{PHT}^{10)}$, or $\mathrm{CBZ}^{12}$, and that $\mathrm{CBZ}$ influences the clonazepam concentration when both drugs are administered intravenously ${ }^{12)}$, suggesting that an interaction at the absorption site was unlikely in the present study.

In conclusion, the present study demonstrated that the serum concentration of clonazepam was positively proportional to the daily dose per weight and negatively affected by the addition of PB, PHT, and CBZ in linear-combination mode. Concomitant administration of these three anticonvulsants was associated with decreases in the clonazepam concentration.

These findings suggest that careful drug monitoring of the clonazepam concentration is needed in patients also receiving or discontinuing $\mathrm{PB}, \mathrm{PHT}$, or $\mathrm{CBZ}$.

\section{References}

1) S.Sato, B.A. Malow, “Antiepileptic Drugs (4th ed.)”,ed.by R.H. Levy, R.H. Mattson, B.S. Meldrum, Raven Press Ltd., New York, 1995, pp. 725-734.

2) A. Berlin, H.Dahlstrom, Eur. J. Clin. Pharmacol., 9, 155-159(1975).

3) F.E.Dreifuss, J.K.Penry, S.W.Rose, H.J.Kupferberg, P.Dyken, S.Sato, Neurology, 25, 255-258 (1975).

4) J. Naestoft, M. Lund, Acta. Neurol. Scandinav., 49, 103-108(1973).

5) O. Sjo, E. F. Hvidberg, J. Naestoft, M. Lund, Eur.J. Clin. Pharmacol., 8, 249-254(1975). 
6) M. Gerna, P. L. Morselli, J. Chromatogr., 116, 445-450(1976).

7) A. Baruzzi, B. Bordo, L. Bossi, D. Castelli, M. Gerna, G. Tognoni, P. Zagnoni, Int. J. Clin. Pharmacol., 15, 403-408(1977).

8) N. Morikawa, K. Ikawa, T. Izumi, M. Takeyama, Jpn. J. Hosp. Pharm., 23, 36-42(1997).

9) J. O. McNamara,"The Pharmacological Basis of Therapeutics ( 9 th ed.)”,ed. By J. G. Hardman, L. E. Limbird, McGraw-Hill Inc., New York, 1996, pp. 461-486.

10) K. C. Khoo, J. Mendels, M. Rothbart, W. A. Garland, W. A. Colburn, B. H. Min, R. Lucek, J. J. Carbone, H. G. Boxenbaum, S. A. Kaplan, Clin, Pharmacol. Ther., 28, 368-375(1980).

11) R. N. Nanda, R. H. Johnson, H. J. Keogh, D.G.Lambie, I.D.Melville, J. Neurol. Neurosurg. Psychiatry, 40, 538-543(1977).

12) A. A. Lai, R. H. Levy, R. E. Cutler, Clin. Pharmacol. Ther., 24, 316-323(1978).

13) W. Sunaoshi, H. Miura, H. Shirai, Jpn. J. Psychiatry Neurol ., 42, 589-591 (1988).

14) L. Gram, K. Wulff, K. E. Rasmussen, Epilepsia, 18, 141-148(1977).

15) W.K. Hildebrandt, Am. J. Hosp. Pharm., 36, 22-27(1979).

16) J.R. Gillette, J.J. Kamm, H.A. Sasame, Mol. Pharmacol ., 4, 541-548(1968). 